\title{
Poluição do ar como fator de risco para a saúde: uma revisão sistemática no estado de São Paulo
}

\author{
STEFFANI NIKOLI DAPPER, ${ }^{I}$ CAROLINE SPOHR ${ }^{I I}$ \\ e ROSELAINE RUVIARO ZANINI III
}

\section{Contextualização}

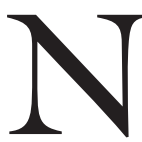

O COMEÇO do século XX, o ar necessário para a respiração de todos os seres vivos da Terra ainda não era abordado de forma tão evidente, pois acreditava-se que este estaria constantemente disponível de forma a manter a vida no planeta (Russo, 2010).

O número crescente da circulação de veículos no mundo e as atividades industriais são fatores que contribuem fortemente para a poluição da atmosfera (Cesar et al., 2013). Esta pode ser originada também por fontes naturais como queima acidental de biomassa (material derivado de plantas ou animais) e erupções vulcânicas (Cançado et al., 2006; Gonçalves et al., 2010).

Novas fontes de poluentes, como a queima de combustíveis fósseis pelos motores, a combustão e a expansão das indústrias siderúrgicas ganharam força com a revolução industrial e isso ocorreu sem um acompanhamento dos possíveis danos que esses poluentes poderiam causar à saúde humana (Coelho, 2007; Mario, 2012).

Esses acontecimentos refletiram em episódios de poluições excessivas, que geraram um aumento no número de mortalidades em algumas cidades da Europa e dos Estados Unidos. Pode-se destacar o mais grave dos episódios ocorrido em 1952, na cidade de Londres, que foi provocado por uma inversão térmica, em que uma quantidade demasiada de poluentes permaneceu sobre a cidade durante três dias, ocasionando um aumento de quatro mil mortes em relação à média do mesmo período (Braga et al., 2001).

Tais episódios impulsionaram estudos na área da Epidemiologia com a finalidade de analisar os efeitos dos poluentes atmosféricos na saúde. A partir disso, diversos países passaram a formular padrões de qualidade do ar, estabelecendo limites de tolerância, os quais garantiriam que a saúde da população não fosse afetada (Martins et al., 2001; Castro et al., 2003; Cançado et al., 2006). 
Apesar de avanços terem surgido nas últimas décadas, em relação a práticas que proporcionem um ar mais limpo, principalmente nos países desenvolvidos, os atuais níveis de poluição atmosférica continuam a ser considerados danosos para a saúde (Gouveia et al., 2006).

Estudos sobre a poluição atmosférica e os efeitos na saúde da população têm demonstrado que, mesmo quando os poluentes se encontram abaixo dos níveis determinados pela legislação, estes são capazes de provocar efeitos na saúde das pessoas (Martins et al., 2002; Moraes et al., 2010; Amâncio; Nascimento, 2012; Gavinier; Nascimento, 2014).

Dentre as faixas etárias mais atingidas pelos efeitos da poluição do ar estão as crianças e os idosos. Pessoas que já sofrem de problemas respiratórios também se tornam mais suscetíveis a sofrer com a elevação nos níveis de poluentes atmosféricos (Martins et al., 2001; Silva et al., 2013).

Além de provocarem efeitos na saúde da população, os problemas causados pela poluição do ar também geram impactos negativos no que se refere à perspectiva econômica e social. Queda da produtividade agrícola, aumento de custos dos sistemas de saúde, maior vulnerabilidade das populações carentes podem ser vistas como exemplos de problemas causados pela contaminação do ar (Instituto de Energia e Meio Ambiente, 2014).

Os efeitos dos poluentes causados ao meio ambiente e à qualidade de vida das pessoas, além de afetarem as comunidades próximas à fonte de emissão, podem viajar milhares de quilômetros pela atmosfera, atingindo locais distantes (Leite et al., 2011).

Os mecanismos pelos quais a poluição do ar interfere na saúde das pessoas ainda não são totalmente conhecidos. Diante disso, estudos epidemiológicos que avaliam essa questão são fundamentais, considerando-se comprovada morbidade respiratória e o efeito negativo que determinados poluentes são capazes de causar na qualidade de vida da população, sendo as hospitalizações apenas um dos efeitos gerados pela degradação do ar (Negrisoli; Nascimento, 2013).

No Brasil, os estudos epidemiológicos analisando a associação de poluentes com problemas na saúde concentram-se no estado de São Paulo. Esta pesquisa teve como objetivo realizar uma revisão sistemática de estudos que avaliaram a relação de doenças com a poluição atmosférica no estado de São Paulo no período de 2010 a 2015 , a fim de verificar quais os poluentes e as doenças mais estudadas, a faixa etária dos indivíduos pesquisados, bem como analisar os resultados das pesquisas quanto a existência ou não da influência dos poluentes na saúde humana.

\section{Poluentes atmosféricos e suas consequências para a saúde}

As estações de monitoramento são responsáveis pelo conhecimento da qualidade do ar. Os equipamentos de medição são caros e requerem grandes estruturas (Jacomino et al., 2009). No estado de São Paulo o órgão responsável pelo monitoramento do ar é a Companhia Ambiental do Estado de São Paulo 
(Cetesb) que realiza a medição sistemática da qualidade do ar. Os poluentes monitorados pela Cetesb servem de indicadores da qualidade do ar e são escolhidos por sua frequência de ocorrência e os seus efeitos adversos. São eles: material particulado (MP), dióxido de enxofre $\left(\mathrm{SO}_{2}\right)$, monóxido de carbono $(\mathrm{CO})$, ozônio $\left(\mathrm{O}_{3}\right)$ compostos orgânicos voláteis $(\mathrm{COV})$ e óxidos de nitrogênio $\left(\mathrm{NO}_{\mathrm{x})}(\right.$ Cetesb, 2015).

Yanagi et al. (2012) salientam que o termo amplo "material particulado" engloba uma série de poluentes tanto sólidos como líquidos que podem ficar suspensos na atmosfera devido ao seu tamanho reduzido.

Estudos epidemiológicos no Brasil têm evidenciado associações de material particulado com a incidência de doenças. Pandya et al. (2002) ressaltam que os gases e partículas ultrafinas provenientes da queima de combustíveis, principalmente do diesel, são fatores responsáveis pela maior incidência de asma brônquica e outras doenças alérgicas em indivíduos.

Gouveia et al. (2006), em seu estudo na cidade de São Paulo (SP), identificaram associação de material particulado inalável ao incremento de 4,6\% nas internações por asma em crianças, de $4,3 \%$ por doença pulmonar obstrutiva crônica em idosos e de $1,5 \%$ por doença isquêmica do coração também em idosos.

Comparado a outros poluentes, o ozônio é o mais complexo e difícil de ser controlado. Este não é emitido diretamente, pois forma-se na baixa atmosfera através de reações fotoquímicas. Os picos de ozônio ocorrem tipicamente em períodos de calor, elevada radiação solar e tempo seco (Fepam, 2014). O estudo de Freitas et al. (2004) encontrou associação entre o aumento de admissões por doenças respiratórias com o ozônio.

Coelho (2007) ressalta que elevadas concentrações de dióxido de nitrogênio $\left(\mathrm{NO}_{2}\right)$ podem provocar problemas respiratórios, especialmente em crianças. Pessoas com asma também podem sofrer com problemas respiratórios adicionais ao entrar em contato com esse poluente.

No que se refere ao dióxido de enxofre $\left(\mathrm{SO}_{2}\right)$, Freitas et al. (2004) ressaltam que esse pode ser facilmente absorvido pelas vias respiratórias superiores, gerando riscos para a saúde. No estudo de Martins et al. (2002) o dióxido de enxofre não ultrapassou os limites aceitáveis dos níveis dos poluentes, mas mesmo assim se mostrou associado aos atendimentos por pneumonia e gripe em idosos. Além disso, Nascimento et al. (2004) encontraram correlação positiva entre dióxido de enxofre com o número de internações por doenças respiratórias.

Coelho (2007) destaca que o monóxido de carbono (CO) é capaz de inibir a troca de oxigênio do sangue com os tecidos vitais e, em concentrações elevadas, pode provocar a morte por envenenamento, afetando principalmente o sistema cardiovascular e nervoso. Martins et al. (2001) encontraram associação de monóxido de carbono com atendimentos por infecções de vias aéreas superiores em São Paulo (SP). 


\section{Metodologia}

Esta pesquisa consiste em uma revisão sistemática desenvolvida com base em estudos sobre a associação da poluição com doenças no estado de São Paulo. Linde e Willich (2003) destacam que a revisão sistemática é uma forma de pesquisa que se baseia na literatura como fonte de dados, sendo considerada capaz de apresentar resultados conflitantes e/ou coincidentes, e proporcionando base para investigações futuras.

Para a obtenção dos artigos a serem analisados, utilizaram-se os descritores "poluição do ar", "qualidade do ar São Paulo" e "poluição do ar São Paulo" na base de dados Scielo (Scientific Eletronic Library Online). O período de pesquisa incluiu estudos publicados de 2010 a 2015.

Primeiramente foram lidos os resumos dos artigos encontrados e assim selecionadas as pesquisas a serem utilizadas na análise. Os critérios de inclusão de artigos foram aqueles publicados em periódicos nacionais e que analisaram a associação da poluição do ar e os efeitos na saúde no estado de São Paulo. Os critérios de exclusão foram artigos que analisaram somente a associação das condições meteorológicas com problemas na saúde, utilizando variáveis como temperatura, umidade, entre outras.

\section{Resultados}

Este tópico apresenta os principais resultados dos estudos referentes à poluição do ar e os efeitos na saúde desenvolvidos no estado de São Paulo.

Foram encontrados no total 92 artigos utilizando os descritores na base de dados Scielo, dos quais 18 se encaixaram nos critérios de inclusão e foram selecionados para a análise.

Na Tabela 1 são apresentados os estudos realizados no período de 2010 a 2015 no estado de São Paulo e publicados em periódicos nacionais.

Ribeiro e Pesquero (2010) desenvolveram seu estudo na cidade de Espírito Santo do Turvo (SP). Os autores investigaram o impacto da queima de cana-de-açúcar na qualidade do ar em uma área sujeita anualmente a esse processo e os efeitos da poluição, em longo prazo, nas condições de saúde respiratória de alunos vivendo na região. Foram comparados os resultados dos questionários obtidos em Espírito Santo do Turvo (SP) com aqueles obtidos em Juquitiba (SP) (cidade onde não é realizada queimada de cana-de-açúcar). Os níveis dos poluentes amostrados, durante a safra de cana-de-açúcar, apresentaram-se abaixo dos padrões recomendados pela legislação brasileira, mesmo assim puderam ser considerados como fatores de risco à saúde respiratória das crianças.

Jasinski et al. (2011) avaliaram os efeitos da poluição em morbidades respiratórias de crianças e adolescentes na cidade de Cubatão (SP). Os resultados mostraram que nas crianças de 0 a 10 anos foram observados os efeitos do material particulado e do ozônio, já nos adolescentes foi verificada associação da poluição com a exposição aguda ao ozônio. 
Tabela 1 - Pesquisas no estado de São Paulo sobre o efeito da poluição do ar na saúde

\begin{tabular}{|c|c|c|c|c|}
\hline Autor & Cidade & Ano & Periódico & Título \\
\hline $\begin{array}{l}\text { Ribeiro e } \\
\text { Pesquero }\end{array}$ & $\begin{array}{l}\text { Espírito } \\
\text { Santo do } \\
\text { Turvo }\end{array}$ & 2010 & $\begin{array}{l}\text { Estudos } \\
\text { Avançados }\end{array}$ & $\begin{array}{l}\text { Queimadas de cana-de-açúcar: avaliação } \\
\text { de efeitos na qualidade do ar e na saúde } \\
\text { respiratória de crianças }\end{array}$ \\
\hline $\begin{array}{l}\text { Jasinski et } \\
\text { al. }\end{array}$ & Cubatão & 2011 & $\begin{array}{l}\text { Cadernos } \\
\text { de Saúde } \\
\text { Pública }\end{array}$ & $\begin{array}{l}\text { Poluição atmosférica e internações } \\
\text { hospitalares por doenças respiratórias } \\
\text { em crianças e adolescentes em Cubatão, } \\
\text { São Paulo, Brasil, entre } 1997 \text { e } 2004\end{array}$ \\
\hline $\begin{array}{l}\text { Amâncio e } \\
\text { Nascimento }\end{array}$ & $\begin{array}{l}\text { São } \\
\text { José dos } \\
\text { Campos }\end{array}$ & 2012 & $\begin{array}{l}\text { Revista da } \\
\text { Associação } \\
\text { Médica } \\
\text { Brasileira }\end{array}$ & $\begin{array}{c}\text { Asma e poluentes ambientais: um estudo } \\
\text { de séries temporais }\end{array}$ \\
\hline $\begin{array}{l}\text { Carneseca } \\
\text { et al. }\end{array}$ & $\begin{array}{l}\text { Ribeirão } \\
\text { Preto }\end{array}$ & 2012 & $\begin{array}{l}\text { Cadernos } \\
\text { de Saúde } \\
\text { Pública }\end{array}$ & $\begin{array}{c}\text { Associação entre a poluição atmosférica } \\
\text { por material particulado e contagens } \\
\text { mensais de procedimentos de inalação } \\
\text { e nebulização em Ribeirão Preto, São } \\
\text { Paulo, Brasil }\end{array}$ \\
\hline $\begin{array}{l}\text { Nascimento } \\
\text { et al. }\end{array}$ & $\begin{array}{l}\text { São } \\
\text { José dos } \\
\text { Campos }\end{array}$ & 2012 & $\begin{array}{l}\text { Cadernos } \\
\text { de Saúde } \\
\text { Pública }\end{array}$ & $\begin{array}{l}\text { Poluentes ambientais e internações } \\
\text { devido a acidente vasculoencefálico }\end{array}$ \\
\hline $\begin{array}{l}\text { Yanagi et } \\
\text { al. }\end{array}$ & São Paulo & 2012 & $\begin{array}{l}\text { Cadernos } \\
\text { de Saúde } \\
\text { Pública }\end{array}$ & $\begin{array}{c}\text { Influência do material particulado } \\
\text { atmosférico na incidência e mortalidade } \\
\text { por câncer no município de São Paulo, } \\
\text { Brasil }\end{array}$ \\
\hline Cesar et al. & Piracicaba & 2013 & $\begin{array}{l}\text { Revista } \\
\text { de Saúde } \\
\text { Pública }\end{array}$ & $\begin{array}{l}\text { Associação entre exposição ao material } \\
\text { particulado e internações por doenças } \\
\text { respiratórias em crianças }\end{array}$ \\
\hline $\begin{array}{l}\text { Nardocci } \\
\text { et al. }\end{array}$ & Cubatão & 2013 & $\begin{array}{l}\text { Cadernos } \\
\text { de Saúde } \\
\text { Pública }\end{array}$ & $\begin{array}{l}\text { Poluição do ar e doenças respiratórias } \\
\text { e cardiovasculares: estudo de séries } \\
\text { temporais em Cubatão, São Paulo, Brasil }\end{array}$ \\
\hline $\begin{array}{l}\text { Nascimento } \\
\text { e Francisco }\end{array}$ & $\begin{array}{l}\text { São } \\
\text { José dos } \\
\text { Campos }\end{array}$ & 2013 & $\begin{array}{l}\text { Cadernos } \\
\text { de Saúde } \\
\text { Pública }\end{array}$ & $\begin{array}{l}\text { Material particulado e internação } \\
\text { hospitalar por hipertensão arterial em } \\
\text { uma cidade brasileira de porte médio }\end{array}$ \\
\hline $\begin{array}{l}\text { Negrisoli e } \\
\text { Nascimento }\end{array}$ & Taubaté & 2013 & $\begin{array}{l}\text { Revista } \\
\text { Paulista de } \\
\text { Pediatria }\end{array}$ & $\begin{array}{l}\text { Poluentes atmosféricos e internações por } \\
\text { pneumonia em crianças }\end{array}$ \\
\hline $\begin{array}{l}\text { Romão et } \\
\text { al. }\end{array}$ & $\begin{array}{l}\text { Santo } \\
\text { André }\end{array}$ & 2013 & $\begin{array}{l}\text { Cadernos } \\
\text { de Saúde } \\
\text { Pública }\end{array}$ & $\begin{array}{l}\text { Relação entre baixo peso ao nascer e } \\
\text { exposição ao material particulado inaláve }\end{array}$ \\
\hline $\begin{array}{l}\text { Amâncio e } \\
\text { Nascimento }\end{array}$ & $\begin{array}{l}\text { São } \\
\text { José dos } \\
\text { Campos }\end{array}$ & 2014 & $\begin{array}{l}\text { São Paulo } \\
\text { Medical } \\
\text { Journal }\end{array}$ & $\begin{array}{l}\text { Poluição ambiental e óbitos devido a } \\
\text { acidente vasculoencefálico em uma } \\
\text { cidade com baixos níveis de poluentes: } \\
\text { estudo ecológico de séries temporais }\end{array}$ \\
\hline $\begin{array}{l}\text { Gavinier e } \\
\text { Nascimento }\end{array}$ & Sorocaba & 2014 & $\begin{array}{l}\text { Ambiente \& } \\
\quad \text { Água }\end{array}$ & $\begin{array}{c}\text { Poluentes atmosféricos e internações por } \\
\text { acidente vascular encefálico }\end{array}$ \\
\hline
\end{tabular}




\begin{tabular}{l|c|c|c|c}
\hline Lima et al. & $\begin{array}{c}\text { São } \\
\text { José dos } \\
\text { Campos }\end{array}$ & 2014 & $\begin{array}{c}\text { Ambiente \& } \\
\text { Água }\end{array}$ & $\begin{array}{c}\text { Associação entre a exposição materna ao } \\
\text { material particulado e parto prematuro }\end{array}$ \\
\hline $\begin{array}{l}\text { Nicolussi } \\
\text { et al. }\end{array}$ & $\begin{array}{c}\text { Ribeirão } \\
\text { Preto }\end{array}$ & 2014 & $\begin{array}{c}\text { Revista } \\
\text { de Saúde } \\
\text { Pública }\end{array}$ & $\begin{array}{c}\text { Poluição do ar e doenças respiratórias } \\
\text { alérgicas em escolares }\end{array}$ \\
\hline $\begin{array}{l}\text { Pinheiro et } \\
\text { al. }\end{array}$ & São Paulo & 2014 & $\begin{array}{c}\text { Revista } \\
\text { de Saúde } \\
\text { Pública }\end{array}$ & $\begin{array}{c}\text { Efeitos isolados e sinérgicos do MP }{ }_{10} \text { e } \\
\text { da temperatura média na mortalidade por } \\
\text { doenças cardiovasculares e respiratórias }\end{array}$ \\
\hline $\begin{array}{l}\text { Santos et } \\
\text { al. }\end{array}$ & $\begin{array}{c}\text { São } \\
\text { Caulsta dos } \\
\text { Campos }\end{array}$ & 2014 & $\begin{array}{c}\text { O papel dos poluentes atmosféricos } \\
\text { sobre o peso ao nascer em cidade de } \\
\text { médio porte Paulista }\end{array}$ \\
\hline $\begin{array}{l}\text { Barbosa } \\
\text { et al. }\end{array}$ & São Paulo & 2015 & $\begin{array}{c}\text { Cadernos } \\
\text { de Saúde } \\
\text { Pública }\end{array}$ & $\begin{array}{c}\text { Poluição do ar e a saúde das crianças: a } \\
\text { doença falciforme }\end{array}$ \\
\hline
\end{tabular}

Amâncio e Nascimento (2012) estimaram o risco para internações por asma em crianças após a exposição de poluentes na cidade de São José dos Campos (SP). Por meio das análises realizadas, foi encontrada forte correlação entre internações com material particulado e dióxido de enxofre. Esses dois poluentes estiveram associados a riscos relativos significativos de 1,01 a 1,04 para internação por asma no mesmo dia e em três dias após a exposição. Quando é aumentada as concentrações desses poluentes, eleva-se o risco de internação em $8 \%$ e $19 \%$, respectivamente. Os autores concluíram que há evidências da influência dos poluentes atmosféricos nas internações por asma na cidade estudada.

Carneseca et al. (2012) analisaram os efeitos do material particulado inalável sobre o número de nebulizações realizadas, considerando-se que esse procedimento pode estar relacionado com eventos adversos à saúde, principalmente se tratando de doenças respiratórias. Com os resultados encontrados, pode-se verificar que o material particulado se mostrou associado ao número de nebulizações realizadas.

O estudo de Nascimento et al. (2012), na cidade de São José dos Campos (SP), buscou estimar os efeitos da poluição atmosférica nas internações por acidente vasculoencefálico. Com os resultados foi verificado que a exposição ao material particulado, no mesmo dia, está associada às internações por acidente vasculoencefálico. Verificou-se também que um acréscimo de $10 \mu \mathrm{g} / \mathrm{m}^{3}$ de material particulado aumenta o risco de internações em $12 \%$. Com base nesses resultados, os autores concluíram que o material particulado no modelo multipoluente está associado a internações por acidente vasculoencefálico.

Yanagi et al. (2012) verificaram a influência do material particulado inalável na incidência e mortalidade por câncer nos distritos em que os poluentes são monitorados em São Paulo (SP). Verificou-se que pele, pulmão, tireoide, laringe e bexiga apresentaram coeficientes de correlação entre 0,60 e $0,80 \mathrm{em}$ alguns períodos para a incidência de câncer. No que se refere aos dados sobre 
a mortalidade, o câncer de pulmão obteve mais correlações nesse intervalo. A análise espacial demonstrou que distritos distantes do centro da cidade de São Paulo apresentaram risco relativo acima do esperado. O estudo revelou que a análise da distribuição espacial dos tipos de câncer em alguns distritos estudados apresentou risco de mortalidade mais elevado do que a de incidência, indicando possíveis deficiências no acesso a diagnósticos e tratamento. Os autores concluíram que o material particulado influencia no aumento da incidência de alguns tipos de câncer contribuindo, consequentemente, para a mortalidade por câncer.

Na cidade de Piracicaba (SP), Cesar et al. (2013) estimaram a associação entre material particulado de 2,5 micra e internações por doenças respiratórias em crianças. Verificou-se que o incremento de $10 \mu \mathrm{g} / \mathrm{m}^{3}$ de material particulado implica o aumento de 7,9 a 8,6 pontos percentuais no risco relativo. Os autores concluíram que a exposição ao material particulado com menos de 2,5 micra mostrou-se associada às internações por doenças respiratórias em crianças.

Nardocci et al. (2013) analisaram a relação da poluição do ar com doenças respiratórias e cardiovasculares verificando associação entre o material particulado com internações para doenças respiratórias totais, doenças respiratórias em menores de 5 anos e doenças cardiovasculares em maiores de 39 anos. Também foi verificada a relação de dióxido de enxofre em maiores de 39 anos e ozônio com doenças cardiovasculares em maiores de 39 anos e doenças respiratórias para menores de 5 anos.

Nascimento e Francisco (2013) avaliaram a associação da exposição das pessoas ao material particulado com internações por hipertensão. Foi verificado que um aumento de $10 \mu \mathrm{g} / \mathrm{m}^{3}$ de material particulado aumenta em 13\% o risco de internações por hipertensão no município de São José dos Campos (SP).

Negrisoli e Nascimento (2013) desenvolveram o primeiro estudo na cidade de Sorocaba (SP) sobre a relação entre poluentes e internações por pneumonia na infância. Foram encontradas fortes correlações entre os poluentes e as internações (com exceção do ozônio). Os autores concluíram que foi possível identificar efeito agudo do dióxido de nitrogênio nas internações por pneumonia, e o efeito do material particulado foi mais tardio, quatro dias após a exposição.

Romão et al. (2013) verificaram que as concentrações de material particulado no terceiro semestre gestacional aumentam o risco de baixo peso ao nascer em 26\% no município de Santo André (SP), ressaltando que os efeitos da poluição vão além de doenças cardiorrespiratórias.

Amâncio e Nascimento (2014) estudaram a associação entre exposição a poluentes e risco de morte por acidente vascular cerebral. O estudo foi desenvolvido na cidade de São José dos Campos (SP) e foi verificada a significância estatística entre a exposição ao material particulado em modelo unipoluente e a importância do material particulado e dióxido de enxofre no modelo multipotente. Percebeu-se que o aumento do risco foi de 10\% a 7\% para material 
particulado e dióxido de enxofre, respectivamente. Concluiu-se que a exposição a poluentes pode ser um fator de risco para morte por acidente vascular cerebral.

Gavinier e Nascimento (2014) estimaram a associação entre poluentes e hospitalizações por acidente vascular encefálico (AVE) em indivíduos com 50 anos de idade ou mais na cidade de Sorocaba (SP), sendo os pioneiros a tratar tal assunto no Brasil. Foi possível identificar associação entre dióxido de nitrogênio e internações por AVE para o terceiro dia de defasagem. Os autores também observaram que o incremento de $10 \mu \mathrm{g} \mathrm{m}^{-3}$ de $\mathrm{NO}_{2}$ representou um aumento de quatro pontos percentuais no risco de internações. Os autores concluíram que o dióxido de nitrogênio está associado a hospitalizações por acidente vascular encefálico em Sorocaba (SP).

Lima et al. (2014) estimaram a relação da exposição de mulheres grávidas ao material particulado e os partos prematuros em São José dos Campos (SP). Os autores concluíram que a exposição da mãe ao material particulado apresenta efeito agudo no nascimento de bebês prematuros. Este estudo foi o primeiro a investigar os efeitos da poluição no parto prematuro no Brasil.

Nicolussi et al. (2014) investigaram a prevalência de doenças respiratórias alérgicas em escolares levando em conta regiões urbanas diferentes e o fluxo de veículos. Os resultados indicaram que onde há intenso tráfego veicular ocorreu a prevalência de asma, rinite e outros sintomas associados.

Pinheiro et al. (2014) analisaram o efeito da poluição atmosférica e da temperatura nos casos de mortalidade por doenças cardiovasculares e respiratórias. Os resultados das pesquisas demonstraram que um aumento de $10 \mu \mathrm{gg} / \mathrm{m}^{3}$ na concentração do material particulado possui um risco relativo de $0,85 \%$ para doenças cardiovasculares e $1,60 \%(0,74 ; 2,46)$ para doenças respiratórias.

Santos et al. (2014) estudaram a associação da exposição materna a poluentes atmosféricos com o baixo peso de bebês ao nascer. Os resultados demonstraram que não foi encontrada associação do baixo peso ao nascer com os poluentes, com exceção do dióxido de enxofre que se mostrou associado no último mês de gestação $(\mathrm{OR}=1,25$; IC95\% 1,00-1,56).

Barbosa et al. (2015) analisaram a associação da poluição com atendimentos de emergência pediátrica de portadores de anemia falciforme verificando que material particulado, dióxido de nitrogênio, dióxido de enxofre, monóxido de carbono e ozônio estiveram associados com um aumento de $18,9 \%, 19 \%, 14,4 \%$, $16,5 \%$ e $9,8 \%$, respectivamente, nos atendimentos totais.

Na Tabela 2 são apresentadas as principais doenças e poluentes estudados nas pesquisas, bem como a faixa etária dos indivíduos.

Pode-se observar, com base nos estudos encontrados, que as principais doenças estudadas no estado de São Paulo no período de 2010 a 2015 se referem, em primeiro lugar, a doenças do trato respiratório em crianças. Tadano (2007) salienta que as crianças são mais suscetíveis aos efeitos da poluição por consumirem o dobro da quantidade de ar dos adultos. 
Em segundo lugar aparecem doenças venosas como acidente vascular cerebral e problemas cardiovasculares. Em terceiro lugar foi estudado o peso dos bebês ao nascer.

Incidência e mortalidade por câncer, partos prematuros e anemia falciforme também foram relacionados com a poluição atmosférica, apresentando resultados positivos na associação com os poluentes estudados.

Tabela 2 - Principais doenças e poluentes estudados no estado de São Paulo (20102015)

\begin{tabular}{|c|c|c|c|}
\hline \multirow{2}{*}{ Autor e ano } & \multicolumn{2}{|c|}{ Variáveis } & \multirow{2}{*}{ Idade } \\
\hline & Doenças & Poluentes & \\
\hline $\begin{array}{l}\text { Ribeiro e } \\
\text { Pesquero, } 2010\end{array}$ & Respiratórias & $\mathrm{PM}_{10}, \mathrm{PTS}^{\star}$ e $\mathrm{NO}_{2}$ & 10 a 13 \\
\hline $\begin{array}{l}\text { Jasinski et al., } \\
2011\end{array}$ & Respiratórias & $\mathrm{PM}_{10}, \mathrm{NO}_{2}, \mathrm{SO}_{2}$ e O & 0 a 19 \\
\hline $\begin{array}{l}\text { Amâncio e } \\
\text { Nascimento, } 2012\end{array}$ & Asma brônquica & $\mathrm{PM}_{10}, \mathrm{O}_{3}, \mathrm{SO}_{2}$ & $\leq 10$ \\
\hline $\begin{array}{l}\text { Carneseca et al., } \\
2012\end{array}$ & $\begin{array}{l}\text { Procedimentos de } \\
\text { inalação/nebulização }\end{array}$ & $\mathrm{MP}_{10}$ & Todas \\
\hline $\begin{array}{l}\text { Nascimento et al., } \\
2012\end{array}$ & $\begin{array}{l}\text { Acidente vascular } \\
\text { cerebral }\end{array}$ & $\mathrm{PM}_{10}, \mathrm{SO}_{2}, \mathrm{O}_{3}$ & $\geq 50$ \\
\hline Yanagi et al., 2012 & $\begin{array}{l}\text { Incidência e } \\
\text { mortalidade por } \\
\text { câncer }\end{array}$ & $\mathrm{MP}_{10}$ & Todas \\
\hline Cesar et al., 2013 & Respiratórias & $\mathrm{MP}_{10}$ & 0 a 10 \\
\hline $\begin{array}{l}\text { Nardocci et al., } \\
2013\end{array}$ & $\begin{array}{l}\text { Respiratórias e } \\
\text { cardiovasculares }\end{array}$ & $\mathrm{PM}_{10}, \mathrm{O}_{3}, \mathrm{SO}_{2}$ & $<5$ e $>39$ \\
\hline $\begin{array}{l}\text { Nascimento e } \\
\text { Francisco, } 2013\end{array}$ & Hipertensão arterial & $\mathrm{PM}_{10}, \mathrm{O}_{3}, \mathrm{SO}_{2}$ & Todas \\
\hline $\begin{array}{l}\text { Negrisoli e } \\
\text { Nascimento, } 2013\end{array}$ & Pneumonia & $\mathrm{MP}_{10}, \mathrm{NO}, \mathrm{NO}_{2} \mathrm{SO}_{2}, \mathrm{O}_{3}$ & 0 a 10 \\
\hline Romão et al., 2013 & Peso ao nascer & $\mathrm{PM}_{10}$ & Recém-nascidos \\
\hline
\end{tabular}




\begin{tabular}{l|c|c|c}
\hline $\begin{array}{l}\text { Amâncio e } \\
\text { Nascimento, 2014 }\end{array}$ & $\begin{array}{c}\text { Acidente vascular } \\
\text { cerebral }\end{array}$ & $\mathrm{MP}_{10}, \mathrm{SO}_{2}, \mathrm{O}_{3}$ & Todas \\
\hline $\begin{array}{l}\text { Gavinier e } \\
\text { Nascimento, 2014 }\end{array}$ & $\begin{array}{c}\text { Acidente vascular } \\
\text { cerebral }\end{array}$ & $\mathrm{MP}_{10}, \mathrm{O}_{3}, \mathrm{NO}, \mathrm{NO}_{2}$ & Recém-nascidos \\
\hline Lima et al., 2014 & $\begin{array}{c}\text { Recém-nascidos } \\
\text { prematuros }\end{array}$ & $\mathrm{MP}_{10}, \mathrm{SO}_{2}, \mathrm{O}_{3}$ & 6 a 7 \\
\hline $\begin{array}{l}\text { Nicolussi et al., } \\
2014\end{array}$ & $\begin{array}{c}\text { Asma, rinite e } \\
\text { eczema atópico }\end{array}$ & $\mathrm{MP}_{10}, \mathrm{SO}_{2}, \mathrm{O}_{3}$ & $>40>60$ \\
\hline $\begin{array}{c}\text { Respiratórias e } \\
\text { cardiovasculares }\end{array}$ & $\mathrm{PM}_{10}$ & Recém-nascidos \\
\hline Santos et al., 2014 & $\begin{array}{c}\text { Peso ao nascer } \\
\text { Barbosa et al., }\end{array}$ & $\mathrm{MP}_{10}, \mathrm{SO}_{2}, \mathrm{O}_{3}$ & $<18$ \\
\hline 2015
\end{tabular}

*Partículas totais em suspensão

\section{Conclusão}

A sociedade vem ampliando gradativamente a importância das responsabilidades sociais e ambientais atreladas aos processos de produção de bens e serviços. Qualquer movimento a favor da formulação de modelos de desenvolvimento mais equilibrados, capazes de sustentar uma relação harmônica entre fatores econômicos, sociais e ambientais, vem se destacando nos últimos anos. Isso é mais relevante considerando as alterações climáticas que vêm ocorrendo em todo o mundo e os seus possíveis reflexos nas condições de vidas no planeta (Batalha et al., 2008).

Todos os estudos inclusos nesta revisão sugerem que existe associação da poluição atmosférica com problemas na saúde das populações estudadas. Uma revisão dos efeitos da poluição do ar e os problemas na saúde realizada por Toledo e Nardocci (2011) na cidade de São Paulo (SP) também encontrou resultados capazes de afirmar que a exposição à poluição atmosférica contribui para o aumento da mortalidade e morbidade, principalmente por doenças respiratórias e cardiovasculares.

Com esta pesquisa pode-se perceber que dentre os estudos desenvolvidos no estado de São Paulo há uma diversidade de doenças estudadas, demonstrando que o efeito da poluição do ar não afeta apenas o sistema respiratório e circulatório da população, mas pode ser causador de risco para outros problemas como o baixo peso ao nascer, incidência e mortalidade por câncer, partos prematuros e anemia falciforme.

No que se refere à idade investigada, a maioria dos estudos avaliou os efeitos da poluição do ar na saúde de crianças, idosos e de recém-nascidos. Percebe-se que poucas são as pesquisas nesta área realizadas com pessoas da faixa etária entre 18 e 64 anos. Sugere-se a investigação dos efeitos dos poluentes nestas 
idades, visto que alguns estudos demonstram a associação de poluentes com problemas na saúde também nessa faixa etária.

No que se refere aos poluentes estudados, a maioria dos trabalhos se limitou a usar os dados disponíveis pelo órgão estadual de monitoramento utilizando, principalmente, os níveis dos seguintes poluentes: monóxido de carbono, ozônio, dióxido de enxofre, dióxido de nitrogênio e material particulado $\left(\mathrm{PM}_{10}\right)$, sendo este último o mais estudado. Entretanto, estudos mais abrangentes deveriam ser realizados para investigar o efeito de outros poluentes, como compostos orgânicos voláteis que também podem ser potenciais causadores de risco para a saúde.

Além disso, com esta revisão foi possível constatar que os estudos desenvolvidos no período de 2010 a 2015 não se limitaram apenas à capital de São Paulo, a qual era o maior alvo dos estudos em períodos anteriores, mas também foram realizados em cidades de médio porte como Cubatão, Espírito Santo do Turvo, Piracicaba, Ribeirão Preto, Santo André, São José dos Campos, Sorocaba e Taubaté.

Por fim, pode-se verificar por meio desta revisão que apesar dos avanços capazes de proporcionar um ar mais limpo, os atuais níveis de poluição continuam a ser danosos para a saúde. Castro et al. (2003) destacam que para que sejam construídas políticas de saúde ambiental eficazes, tornam-se necessários estudos que mostrem a relação entre poluição do ar e a saúde, pois estes podem garantir uma melhoria da qualidade de informação a ser utilizada pelos gestores.

Ressalta-se também a importância de estudos que tratem dos efeitos da interação das pessoas no meio ambiente, pois estes ampliam a possibilidade de adoção de medidas preventivas capazes de diminuir a degradação ambiental e, consequentemente, os efeitos nocivos à saúde das pessoas.

Este estudo será ampliado para investigar outras pesquisas que foram realizadas nos demais estados do Brasil e em outros países, relacionando os efeitos da poluição do ar com a saúde de populações.

\section{Referências}

AMÂNCIO, T. C.; NASCIMENTO, C. F. L. Asma e poluentes ambientais: um estudo de séries temporais. Revista da Associação Médica Brasileira, v.58, n.3, p.302-7, 2012.

AMÂNCIO, T. C.; NASCIMENTO, C. F. L. Poluição ambiental e óbitos devido a acidente vasculoencefálico em uma cidade com baixos níveis de poluentes: estudo ecológico de séries temporais. São Paulo Medical Journal, v.132, n.6, 2014.

BARBOSA, S. M. M. et al. Poluição do ar e a saúde das crianças: a doença falciforme. Cadernos de Saúde Pública, Rio de Janeiro, v.31, n.2, p.265-75. 2015.

BATALHA, O. M. (Coord.) Introdução à engenbaria de produção. Rio de Janeiro: Elsevier, 2008. 
BRAGA, A. et al. Poluição atmosférica e saúde humana. Revista USP, São Paulo, n.5, p.58-71, set./nov. 2001.

CANÇADO, J. E. D. et al. Repercussões clínicas da exposição à poluição atmosférica. Jornal Brasileiro de Pneumologia, v.32, n.Supl 1, p.S5-S1 1, 2006.

CARNESECA, C. E.; ACHCAR, A. J.; MARTINEZ, Z. E. Associação entre a poluição atmosférica por material particulado e contagens mensais de procedimentos de inalação e nebulização em Ribeirão Preto, São Paulo, Brasil. Cadernos de Saúde Pública, Rio de Janeiro, v.28, n.8, p.1591-8, 2012.

CASTRO, A. H.; GOUVEIA, N.; CEJUDO, E. A. J. Questões metodológicas para a investigação dos efeitos da poluição do ar na saúde. Revista Brasileira de Epidemiologia, v.6, n.2, 2003.

CESAR, G. C. A.; NASCIMENTO, C. F. L.; CARVALHO, A. J. Associação entre exposição ao material particulado e internações por doenças respiratórias em crianças. Revista de Saúde Pública, v.47, n.6, p.1209-12, 2013.

CETESB. Poluentes. Disponível em: <http://ar.cetesb.sp.gov.br/poluentes/> Acesso em: 28 nov. 2015.

COELHO, S. Z. S. M. Uma análise estatística com vistas a previsibilidade de internações por doenças respiratórias em função de condições meteorotrópicas na cidade de São Paulo. 2007. Tese (Doutorado em Ciências) - Universidade de São Paulo. São Paulo, 2007.

FEPAM. Diagnóstico da Qualidade do Ar no Rio Grande do Sul no período de 2003 a 2012. 2014. Disponível em: <http://www.fepam.rs.gov.br/qualidade/relatorio_anual_auto.asp?id=A.A\&status=s $>$. Acesso em: 17 nov. 2015.

FREITAS, C. et al. Internações e óbitos e sua relação com a poluição atmosférica em São Paulo, 1993 a 1997. Revista Saúde Pública, v.38, n.6, p.751-7, 2004.

GAVINIER, S.; NASCIMENTO, C. F. L. Poluentes atmosféricos e internações por acidente vascular encefálico. Ambiente \& Água - An Interdisciplinary Journal of Applied Science, Taubaté, v.9, n.3, jul./sep. 2014.

GONÇALVES, T. L. F. et al. Modelagem dos processos de remoção sulfato e dióxido de enxofre presente no particulado em diferentes localidades da região metropolitana de São Paulo. Revista brasileira de Geofísica, v.28, p.109-19, 2010.

GOUVEIA, N. et al. Hospitalizações por causas respiratórias e cardiovasculares associadas à contaminação atmosférica no Município de São Paulo. Cadernos de Saúde Pública, Rio de Janeiro, v.22, n.12, p.2669-77, 2006.

INSTITUTO DE ENERGIA E MEIO AMBIENTE. $1^{\circ}$ Diagnóstico da rede de monitoramento da qualidade do ar no Brasil. 2014. Disponível em: <http://www.forumclima. pr.gov.br/arquivos/File/Rosana/Diagnostico_Qualidade_do_Ar_Versao_Final_Std. pdf> Acesso em: 15 jun. 2015.

JACOMINO, F. M. V. et al. Avaliação da qualidade do ar em um polo produtor de ferro-gusa. Engenharia Sanitária e Ambiental. v.14, n.4, p.511-20, out./dez. 2009.

JASINSKI, R.; PEREIRA, L. A. A.; BRAGA, A. L. F. Poluição atmosférica e internações hospitalares por doenças respiratórias em crianças e adolescentes em Cubatão, São 
Paulo, Brasil, entre 1997 e 2004. Cadernos de Saúde Pública, Rio de Janeiro, v.27, n.11, p.2242-52, 2011.

LEITE, R. C. M. et al. Utilização de regressão logística simples na verificação da qualidade do ar atmosférico de Uberlândia. Engenharia Sanitária e Ambiental, v.16, n.1, jan./mar. 2011.

LIMA, C. A. T. et al. Associação entre a exposição materna ao material particulado e parto prematuro. Ambiente \& Água - An Interdisciplinary Journal of Applied Science, Taubaté, v.9, n.1, jan./mar. 2014.

LINDE, K.; WILLICH, S. N. How objective are systematic reviews? Differences between reviews on complementary medicine. Journal of the Royal Society of Medicine, v.96, p.17-22, 2003.

MARIO, M. P. J. Poluição atmosférica como condicionante no processo de ocupação do espaço urbano: Análise na cidade de Porto Alegre, RS. 2012. Dissertação (Mestrado em Planejamento Urbano e Industrial) - Universidade Federal do Rio Grande do Sul. Porto Alegre, 2012

MARTINS, L. C. et al. Relação entre poluição atmosférica e atendimentos por infecção de vias aéreas superiores no município de São Paulo: avaliação do rodízio de veículos. Revista Brasileira de Epidemiologia, v.4, n.3, p.220-9, 2001.

MARTINS, L. C. et al. Poluição atmosférica e atendimentos por pneumonia e gripe em São Paulo, Brasil.. Revista de Saúde Pública, v.36, n.1, p.88-94. 2002.

MORAES, A. C. L. et al. Sibilância em crianças e adolescentes vizinhos a uma indústria petroquímica no Rio Grande do Norte, Brasil. Jornal de Pediatria, v.86, n.4, p.337-44, 2010.

NARDOCCI, A. C. et al. Poluição do ar e doenças respiratórias e cardiovasculares: estudo de séries temporais em Cubatão, São Paulo, Brasil. Cadernos de Saúde Pública, Rio de Janeiro, v.29, v.9, p.1867-76, 2013.

NASCIMENTO, L. F. C. Poluentes ambientais e internações devido a acidente vasculoencéfalico. Cadernos de Saúde Pública, Rio de Janeiro, v.28, n.7, p.1319-24, 2012.

NASCIMENTO, L. F. C.; FRANCISCO, J. B. Material particulado e internação hospitalar por hipertensão arterial em uma cidade brasileira de porte médio. Cadernos de Saúde Pública, Rio de Janeiro, v.29, n.8, p.1565-71, 2013.

NASCIMENTO, L. F. C.; MÓDOLO, C. C. M.; JUNIOR, C. A. J. Efeitos da poluição atmosférica na saúde infantil: um estudo ecológico no Vale do Paraíba. Revista Brasileira de Saúde Materna e Infantil, Recife, v.4, n.4, p.367-74, out./dez. 2004.

NEGRISOLI. J.; NASCIMENTO. C. F. L. Poluentes atmosféricos e internações por pneumonia em crianças. Revista Paulista de Pediatria, v.31, n.4, p.501-6, 2013.

NICOLUSSI, F. H. et al. Poluição do ar e doenças respiratórias alérgicas em escolares. Revista de Saúde Pública, v.48, n.2, p.326-30, 2014.

PANDYA, R. J. et al. Diesel exhaust and asthma: hypotheses and molecular mechanism of action. Environ Health Perspect, v.110, (suppl 1), p.103-12, 2002.

PINHEIRO, S. L. L. A. et al. Efeitos isolados e sinérgicos do MP10 e da temperatura média na mortalidade por doenças cardiovasculares e respiratórias. Revista de Saúde Pública, v.48, n.6, p.881-8, 2014. 
RIBEIRO, H.; PESQUERO, C. Queimadas de cana-de-açúcar: avaliação de efeitos na qualidade do ar e na saúde respiratória de crianças. Estudos Avançados, v.24, n.68, 2010.

ROMÃO, R. et al. Relação entre baixo peso ao nascer e exposição ao material particulado inalável. Cadernos de Saúde Pública, Rio de Janeiro, v.29, n.6, p.1101-8, 2013.

RUSSO, P. R. A qualidade do ar no município do Rio de Janeiro : análise espaço-temporal de partículas em suspensão na atmosfera. Revista de Ciências Humanas, v.10, n.1, p.78-93, jan./jun. 2010.

SANTOS, V. P. et al. O papel dos poluentes atmosféricos sobre o peso ao nascer em cidade de médio porte Paulista. Revista Paulista de Pediatria, v.32, n.4, p.306-12, 2014.

SILVA, C. M. A. et al. Material particulado originário de queimadas e doenças respiratórias. Revista de Saúde Pública, v.47, n.2, p.345-52, 2013.

TADANO, S. Y. Análise do impacto de MP10 na saúde populacional: estudo de caso em Araucária, PR. 2007. Dissertação (Mestrado em Engenharia Mecânica) - Universidade Tecnológica Federal do Paraná. Curitiba, 2007.

TOLEDO, G. I. F. M.; NARDOCCI, A. C. Poluição veicular e saúde da população: uma revisão sobre o município de São Paulo (SP), Brasil. Revista Brasileira de Epidemiologia, v.14, n.3, p.445-54, 2011.

YANAGI, Y.; ASSUNÇÃO, V. J.; BARROZO, V. L. Influência do material particulado atmosférico na incidência e mortalidade por câncer no Município de São Paulo, Brasil. Cadernos de Saúde Pública, Rio de Janeiro, v.28, n.9, p.1737-48, 2012.

RESUMO - Além de prejudicar a fauna e a flora, a poluição atmosférica é capaz de afetar a saúde das pessoas. Este estudo teve como objetivo realizar uma revisão sistemática dos estudos epidemiológicos desenvolvidos no estado de São Paulo que avaliaram os efeitos da poluição do ar na saúde da população. Todas as pesquisas encontradas sugerem que existe associação da poluição atmosférica com problemas na saúde das populações estudadas. Dentre os principais trabalhos desenvolvidos, há uma diversidade de doenças estudadas, demonstrando que o efeito da poluição do ar não afeta apenas o sistema respiratório, mas pode ser causador de risco para outros problemas como o baixo peso ao nascer, incidência e mortalidade por câncer, partos prematuros e anemia falciforme.

PALAVRAS-CHAVE: Qualidade do ar, Poluição ambiental, Poluentes atmosféricos.

ABSTRACT - In addition to damaging flora and fauna, air pollution can affect people's health. This paper is a systematic review of epidemiological studies carried out in the state São Paulo assessing the effects of air pollution on health, all of them suggesting an association between atmospheric pollution and health problems in the population. Several diseases were studied, demonstrating that air pollution not only affects the res- 
piratory system, but can also be a risk factor for other problems, such as low weight at birth, incidence of and mortality from cancer, premature births and sickle cell anemia. KEYWORDS: Air quality, Environmental pollution, Air pollutants.

Steffani Nikoli Dapper é mestranda no Programa de Pós-Graduação em Engenharia de Produção da Universidade Federal de Santa Maria (UFSM), Rio Grande do Sul. @ - stenikoli@hotmail.com

Caroline Spohr é especialista em Estatística e Modelagem Quantitativa pela Universidade Federal de Santa Maria (UFSM), Rio Grande do Sul.

$@$ - caroline.spohr@yahoo.com

Roselaine Ruviaro Zanini é doutora em Epidemiologia pela Universidade Federal do Rio Grande do Sul (UFRGS). @ - rrzanini63@gmail.com

Recebido em 17.12.2015 e aceito em 17.2.2016.

I, II Universidade Federal de Santa Maria. Santa Maria/Rio Grande do Sul, Brasil.

III Universidade Federal do Rio Grande do Sul. Porto Alegre/Rio Grande do Sul, Brasil. 
